\title{
COMMENTARY/
}

\author{
by Bernard Dixon
}

\section{EXPLOITING SECONDARY METABOLISM OF MICROBES}

W

as Darwin wrong? Do new species appear gradually, or suddenly by "punctuated equilibrium?" Does Lamarckian inheritance operate in the immune system? Is the natural selection of novelties, generated by capricious accidents, really sufficient to account for the whole, rich profusion of life on earth?

The most arcane scientific controversy to have received public coverage in recent years must be that over evolution. And one of the oddest features of the debate is that, while arguing over many esoteric issues, its protagonists have ignored an even greater perplexity: the secondary metabolism of microorganisms. For here we have a phenomenon as difficult to square with ruthless Darwinian selection as those more favoured puzzles, the evolution of the peacock's tail or the emergence of altruistic behaviour. How to explain why certain microbes expend energy and materials in synthesizing, often via multi-enzyme complexes, substances with highly elaborate structures but without any clearly recognizable function for the producer cells?

Biotechnologists have a particular interest in this riddle-and not merely for its intellectual fascination. Having stumbled on secondary metabolism by chance, because one category of its products (the antibiotics) is medically invaluable, they are now discerning signs of a multitude of further metabolites. Useless to the producers, but useful to man, vast numbers of these potential treasures await only the development of techniques sufficiently sensitive and ingenious to demonstrate their existence.

One man who has done more than most to wrestle with the puzzle of secondary metabolism, and point the way to its more concerted exploitation in the future, is Hans Zähner of the University of Tübingen, West Germany. He likens this apparently wasteful but novelty-generating machinery to a "games room," possessed by some organisms, in which they can "try out" new biochemical strategies. But these are insulated from the lethal penalty which awaits unsuccessful experimentation at the level of intermediary metabolism, regulation, transport, differentiation or morphogenesis. It is rather like using a large note pad to tinker with improvements in Shakespeare's Hamlet-as against altering complete texts of the play and discarding every failure, which would be grotesquely wasteful of paper.

Writing recently in Bioactive Microbial Products: Search and Discovery (edited by J. D. Bu'Lock et al, Academic Press), Professor Zähner coupled this picture of elbow-room in biochemical evolution with a more speculative view that there is a division of labour among microorganisms. Thus a novel synthetic capacity

Bernard Dixon, Ph.D., is a microbiologist and regular columnist for BIO/ TECHNOLOGY. He is a former editor of New Scientist. arising in a streptomycete (a group with multifarious secondary metabolism) could be passed on genetically not only to its descendents but also to other organisms. It's possible, through plasmids, phages, protoplast fusion, and other routes, that transfers of this sort allow many new compounds to find functions in cells quite unrelated to those in which they originated.

A persuasive example is the $\beta$-lactams. There seem, today, to be many more streptomycetes than fungi which produce these antibiotics. Could it be that Cephalosporium did not itself develop machinery for synthesizing cephalosporins, but acquired the relevant information from a streptomycete? And could the curiously unequal distribution of secondary metabolites among different microbial groups reflect not only the size of their corresponding "games rooms" but also restrictions (by endonucleases?) on such uptakes of genetic material?

Professor Zähner has now pushed this interpretation so far as to hypothesize that the immense variety of secondary metabolism is a necessity, or even a prerequisite, for the continuance of biochemical evolution. Compared with the potentially disastrous consequences of unsuccessful experiments in areas such as intermediary metabolism and regulation, which are exposed immediately to the scythe of Darwinian selection, secondary metabolism allows an infinitely greater number of possibilities to be tried out. Add to this a widespread horizontal transmission of information, and you have a scenário at odds with, and incomparably richer than, that of Darwinian evolution.

But what are the practical implications of this newly emerging view of microbial biochemistry? The broad answer is a rapid demise of the conventional opinion, held firmly up until just a few years ago that we are unlikely to discover many further new and useful metabolites. Several reports have already confirmed that, given confidence that such unknowns actually exist plus skill in prospecting for them, they will indeed be found. One technique being pioneered by Zähner and his colleagues is the use of ethylenediaminetetraacetic acid to increase cell permeability and thereby expose substances unable to penetrate whole cells. There is, after all, no reason to work within the physical constraints imposed by nature.

Professor Zähner's most radical suggestion, however, is that we should move far away from conventional screening procedures, abandoning microbial tests as the first move and replacing them by thin layer chromatography. Even more efficient would be "a combination of high-pressure liquid chromatography with an as-yet-to-be-developed carbon detector and mass spectrometer." Using such a sequence, he believes, every single one of the secondary metabolites generated by a particular strain could be revealed and captured for biological scrutiny. From Zähner's preliminary work with TLC, it appears Continued on page 211 
BIO/TECHNOLOGY (continued from page 137)

ary, cooperative advance between man and other life forms which biotechnology represents and counter the concept of natural disruption now associated with other technologies, especially nuclear technologies. To reinforce this effort, the tradition of fermentation, its safe use in the wine, cheese, and pharmaceutical industries, and its link to the new technologies should be introduced as new fermentation products are introduced. The stability, the safety, and the reliability of such procedures should also be stressed.

An industrial pre-marketing consumer education effort should also emphasize aspects of biotechnology which counter some of the current prejudices against other technologies. Consumers who rightly fear acid rain and meltdowns may be less reluctant to accept biotechnology products when the "clean" aspects of these processes are emphasized. The potentials for substituting enzymatic reactions for toxin-producing chemical reactions, the ability to develop non-polluting alternative energy forms, and the development of environmental monitoring products can be used as potent contrasts to the current view of technological evils.

The network for preparing consumers for biotechnology already exists in the marketing and public relations departments of corporations. The multimillion dollar budgets for reaching consumers through media and educational programs also exist. If corporations decide to use these resources with the same foresight and strategic planning which has characterized biotechnology research, the public can be prepared to accept and benefit from the proliferation of new products which will be introduced in the forseeable future.-Christopher Edwards

COMMENTARY (continued from page 174)

that the chances of finding something interesting would be greater with only 100 new compounds screened chemically and given biological tests, than with 10000 microbial strains examined by traditional techniques.

"The theory determines what one observes," Albert Einstein once said. The verdict of history may well be that Hans Zähner is the man who, during the 1980s, altered the whole course of applied microbiology by crucially altering those rules of observation.

FINAL WORD (continued from page 212)

and practices of biotechnology and provides an insight into how, from the perspective of their individual disciplines, students will best be able to contribute to progress in the field. As a third possibility, the certificate program might arrange for students to spend time working in the laboratories of biotechnology companies where they will gain respect for applied science.

Certificate programs are not without problems. However, they are not disruptive to traditional departmental authority, nor do they widen the division between members of the program and their colleagues who do not participate in the program. They require no major changes in the operation or organization of university administrative structures, and certificate programs have the additional advantage in that they self destruct when interest in the field diminishes or disappears.

The system recommended here of a separately organized center and a certificate program in biotechnology complements the activities of traditional departments and offers the advantage of perpetuating a close association of research and teaching which is characteristic of quality higher education in America. It already has been implemented by some leading universities and is deserving of serious consideration by others intending to capitalize on the opportunity biotechnology has created.

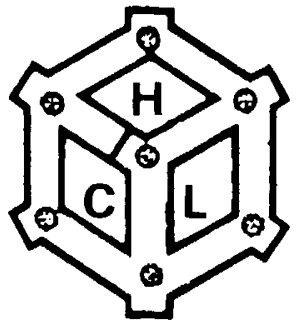

\section{RESEARCH \& DEVELOPMENT SERVICES in BIOTECHNOLOGY}

Since 1938 Haldane Consultants have been providing a research and development service in what is now widely known as biotechnology. A wide range of projects are progressed in their modern well equipped laboratories. Examples of projects are:-

Feasibility studies.
Development of process from inception to producing sufficient material for clinical or commercial evaluation.

\section{Assessing potential uses of raw materials and organic waste materials.}

\section{Process verification.}

Extraction and isolation.

The facilities include sterile product facilities, Category A containment and culture facilities for microorganisms, algae, animal cells and Plant cells in both batch and continuous culture systems

Enquiries about specific projects should be addressed to the Director:

\author{
Haldane Consultants Limited, \\ 40, Nuffield Road, \\ Poole, Dorset BH17 7RA. \\ England.
}

Tel. Poole (0202) 678037

Circle No. 78 on Reader Service Card 